PROCEEDINGS OF THE

AMERICAN MATHEMATICAL SOCIETY

Volume 132, Number 10, Pages 3047-3053

S 0002-9939(04)07406-4

Article electronically published on May 12, 2004

\title{
ON ALLEE EFFECTS IN STRUCTURED POPULATIONS
}

\author{
SEBASTIAN J. SCHREIBER
}

(Communicated by Carmen C. Chicone)

\begin{abstract}
Maps $f(x)=A(x) x$ of the nonnegative cone $C$ of $\mathbf{R}^{k}$ into itself are considered where $A(x)$ are nonnegative, primitive matrices with nondecreasing entries and at least one increasing entry. Let $\lambda(x)$ denote the dominant eigenvalue of $A(x)$ and $\lambda(\infty)=\sup _{x \in C} \lambda(x)$. These maps are shown to exhibit a dynamical trichotomy. First, if $\lambda(0) \geq 1$, then $\lim _{n \rightarrow \infty}\left\|f^{n}(x)\right\|=\infty$ for all nonzero $x \in C$. Second, if $\lambda(\infty) \leq 1$, then $\lim _{n \rightarrow \infty} f^{n}(x)=0$ for all $x \in C$. Finally, if $\lambda(0)<1$ and $\lambda(\infty)>1$, then there exists a compact invariant hypersurface $\Gamma$ separating $C$. For $x$ below $\Gamma$, $\lim _{n \rightarrow \infty} f^{n}(x)=0$, while for $x$ above, $\lim _{n \rightarrow \infty}\left\|f^{n}(x)\right\|=\infty$. An application to nonlinear Leslie matrices is given.
\end{abstract}

\section{INTRODUCTION}

Difference equation models for single species population dynamics are of the form

$$
x^{\prime}=a(x) x
$$

where $x$ is a nonnegative real representing the current population density, $a(x)$ is the per-capita growth rate of the population, and $x^{\prime}$ is the population density in the next generation. When resources are abundant, the per-capita growth rate $a(x)$ can be an increasing function due to predator saturation, antipredator vigilance or aggression, cooperative predation or resource defense, increased availability of mates, and conspecific enhancement of reproduction [3, 8, 9. For example, Hassell [4] in modeling a population subject to predation by a satiating generalist uses $a(x)=\lambda \exp (-\alpha /(1+\beta x))$ where $\lambda$ is the geometric growth rate of the population in the absence of predation, $\alpha$ determines the maximum predation rate, and $\beta$ determines how quickly the predators satiate. Models of this type are easily seen to exhibit a trichotomy of dynamical behaviors:

- If $a(0) \geq 1$, then $\lim _{n \rightarrow \infty} f^{n}(x)=\infty$ for all $x>0$.

- If $\lim _{x \rightarrow \infty} a(x) \leq 1$, then $\lim _{n \rightarrow \infty} f^{n}(x)=0$ for all $x \geq 0$.

- If $\lim _{x \rightarrow \infty} a(x)>1$ (possibly infinite) and $a(0)<1$, then there exists a positive equilibrium $x^{*}$ such that $\lim _{n \rightarrow \infty} f^{n}(x)=0$ for all $x<x^{*}$ and $\lim _{n \rightarrow \infty} f^{n}(x)=\infty$ for all $x>x^{*}$.

The last of these dynamical behaviors corresponds to what is known as a (strong) Allee effect - there exists a critical density below which extinction is inevitable [8].

Received by the editors March 17, 2003 and, in revised form, May 20, 2003.

2000 Mathematics Subject Classification. Primary 37N25, 92D25, 37C65.

This research was supported in part by National Science Foundation Grant DMS-0077986.

(C)2004 American Mathematical Society 
The goal of this article is to extend the dynamical trichotomy from onedimensional maps for unstructured populations to $k$-dimensional maps for structured populations (i.e., populations divided up into subpopulation via age, stage, or space). The remainder of the article is structured as follows. In section 2 , we present the multi-dimensional analogs of (1), introduce basic definitions, and state the main result. The main result is proven in section 3 , and an application to age-structured populations is given in section 4 .

\section{MAIN RESULt}

Let $C$ denote the nonnegative cone of $\mathbf{R}^{k}$. Given $x=\left(x_{1}, \ldots, x_{k}\right)$ and $y=$ $\left(y_{1}, \ldots, y_{k}\right)$ in $C$, we write $x \leq y$ if $x_{i} \leq y_{i}$ for all $i, x<y$ if $x \leq y$ and $x \neq y$, and $x \ll y$ if $x_{i}<y_{i}$ for all $i$. Given two $k \times k$ matrices, $A$ and $B$, whose $(i, j)$-th entries are given by $a_{i j}$ and $b_{i j}$, we write $A \leq B$ if $a_{i j} \leq b_{i j}$ for all $i$ and $j, A<B$ if $A \leq B$ and $A \neq B$, and $A \ll B$ if $a_{i j}<b_{i j}$ for all $i$ and $j$. Let $f: C \rightarrow C$ be given by

$$
f(x)=A(x) x
$$

where $A(x)$ are $k \times k$ nonnegative matrices whose $(i, j)$-th entries are given by $a_{i j}(x)$. About $A(x)$ we make the following assumptions:

A1: $A(0)$ is primitive (i.e., there exists a positive integer $n$ such that all the entries of $A(0)^{n}$ are positive),

A2: $x \mapsto A(x)$ is continuously differentiable,

A3: $A(x) \leq A(y)$ whenever $x \leq y$, and

A4: there exist $i, j, l$ such that for all $x \in C, \frac{\partial a_{i j}}{\partial x_{l}}(x)>0$.

Define the limiting matrix $A(\infty)$ with entries $a_{i j}(\infty)$ by

$$
a_{i j}(\infty)=\sup _{x \in C} a_{i j}(x) \text {. }
$$

Some of the entries of this matrix may be $\infty$. Assumptions A1 and A3 imply that $A(x)$ is primitive for all $x \in C$. Hence (see, e.g., [7]), there exists a dominant eigenvalue $\lambda(x)>0$. Define $\lambda(\infty)$ to be the dominant eigenvalue of $A(\infty)$ if all the entries of $A(\infty)$ are finite, and $\infty$ otherwise. Given a point $x \in C$, let

$$
\|x\|=\max \left\{x_{1}, \ldots, x_{k}\right\}, \quad \min (x)=\min \left\{x_{1}, \ldots, x_{k}\right\} .
$$

Our main result is:

Theorem 1. $f(x)$ has three possible dynamics:

(1) If $\lambda(\infty) \leq 1$, then $\lim _{n \rightarrow \infty} f^{n}(x)=0$ for any $x \in C$.

(2) If $\lambda(0) \geq 1$, then $\lim _{n \rightarrow \infty} \min \left(f^{n}(x)\right)=\infty$ for any nonzero $x \in C$.

(3) If $\lambda(0)<1$ and $\lambda(\infty)>1$, then there exists a continuously differentiable invariant hypersurface $\Gamma$ that separates $C$ into two pieces. For points $x$ below $\Gamma$ (i.e., $x$ such that $x<y$ for some $y \in \Gamma$ ),

$$
\lim _{n \rightarrow \infty} f^{n}(x)=0 \text {. }
$$

Alternatively, for points $x$ above $\Gamma$ (i.e., $x$ such that $x>y$ for some $y \in \Gamma$ ),

$$
\lim _{n \rightarrow \infty} \min \left(f^{n}(x)\right)=\infty .
$$

Remark. A similar result can be proven for ordinary differential equations of the form $\frac{d x}{d t}=A(x) x$ where $A(x)$ are $k \times k$ matrices (not necessarily nonnegative), $\exp (A(0))$ is primitive, and assumptions $\mathbf{A 2}-\mathbf{A} 4$ hold. 


\section{Proof of MAIN RESUlt}

The proof of Theorem 1 relies heavily on the fact that $f(x)$ is a monotone map (i.e., $f(x) \geq f(y)$ whenever $x \geq y$ ). In fact, the following lemma implies that there exists a positive integer $N$ such that $f^{N}(x)$ is strongly monotone (i.e., $f^{N}(x) \gg f^{N}(y)$ whenever $\left.x>y\right)$.

Lemma 1. $A(x)$ has the following properties:

(1) There exists a positive integer $N$ such that the entries of $A(0)^{N}$ are all positive and $A(x)^{N} \gg A(y)^{N}$ whenever $x \gg y$.

(2) $\lambda(x) \geq \lambda(y)$ whenever $x \geq y$.

(3) $\lambda(x)>\lambda(y)$ whenever $x \gg y$.

Proof. Since $A(0)$ is primitive, there exists a positive integer $L$ such that all the entries of $A(0)^{L}$ are positive. Set $N=3 L$. To see why this gives the desired $N$, assume $x \gg y$. Assumptions A3 and A4 imply that $A(x)^{L}>A(y)^{L}>A(0)^{L}$. In particular, all the entries of $A(y)^{L}$ are positive and one of the entries of $A(x)^{L}$ is greater than the corresponding entry of $A(y)^{L}$. It follows that all the entries of a row and a column of $A(x)^{2 L}$ are greater than the corresponding entries of $A(y)^{2 L}$. Iterating one more time yields that $A(x)^{3 L} \gg A(y)^{3 L}$.

Assume $x \geq y$. Since $A(x) \geq A(y)$,

$$
\lambda(x)=\lim _{n \rightarrow \infty}\left\|A(x)^{n}\right\|^{\frac{1}{n}} \geq \lim _{n \rightarrow \infty}\left\|A(y)^{n}\right\|^{\frac{1}{n}}=\lambda(y) .
$$

Assume $x \gg y$. Since $A(x)^{N} \gg A(y)^{N}$, there exists an $\epsilon>0$ such that $A(x)^{N} \geq$ $(1+\epsilon)^{N} A(y)^{N}$. Thus $\lambda(x) \geq(1+\epsilon) \lambda(y)$.

Assume $\lambda(\infty) \leq 1$. Choose an $x \in C$. Since $\lambda(\infty) \leq 1$ and $A(\infty)$ is primitive, the Perron-Frobenius Theorem (see, e.g., [7]) implies that there exists a constant $c \geq 1$ such that $\left\|A(\infty)^{n}\right\| \leq c$ for all $n \geq 0$. Since $A(y) \leq A(\infty)$ for all $y \in C$,

$$
\left\|f^{n}(x)\right\|=\left\|A\left(f^{n-1}(x)\right) \ldots A(x) x\right\| \leq\left\|A(\infty)^{n} x\right\| \leq c\|x\|
$$

for all $n \geq 0$. Let $z=c\|x\|(1,1, \ldots, 1)$. Lemma 1 implies that $\lambda(z)<\lambda(\infty) \leq 1$. Since $f^{n}(x) \leq z$ for all $n \geq 0$, we have that $A\left(f^{n}(x)\right) \leq A(z)$ for all $n \geq 0$. The Perron-Frobenius Theorem implies that $\lim _{n \rightarrow \infty}\left\|A(z)^{n} v\right\|^{\frac{1}{n}}=\lambda(z)$ for all $v>0$. Therefore,

$$
\limsup _{n \rightarrow \infty}\left\|f^{n}(x)\right\|^{1 / n}=\limsup _{n \rightarrow \infty}\left\|A\left(f^{n-1}(x)\right) \ldots A(x) x\right\|^{1 / n} \leq \lambda(z)<1 .
$$

In particular, $\lim _{n \rightarrow \infty} f^{n}(x)=0$.

Next, assume $\lambda(0) \geq 1$. Choose $x \gg 0$. Since $\lambda(0) \geq 1$ and $A(0)$ is primitive, the Perron-Frobenius Theorem implies that there exists a constant $c>0$ such that $A(0)^{n} x \geq c x$ for all $n \geq N$. Since $A(y) \geq A(0)$ for all $y \in C$,

$$
f^{n}(x)=A\left(f^{n-1}(x)\right) \ldots A(x) x \geq A(0)^{n} x \geq c x
$$

for all $n \geq N$. By Lemma 1 $\lambda(c x)>\lambda(0) \geq 1$. Since $f^{n}(x) \geq c x$ for all $n \geq N$, we have $A\left(f^{n}(x)\right) \geq A(c x)$ for all $n \geq N$. The Perron-Frobenius Theorem implies that $\lim _{n \rightarrow \infty} \min \left(A(c x)^{n} v\right)^{\frac{1}{n}}=\lambda(c x)$ for all $v \in C$. Hence

$$
\liminf _{n \rightarrow \infty} \min \left(f^{n}(x)\right)^{1 / n}=\liminf _{n \rightarrow \infty} \min \left(A\left(f^{n-1} x\right) \ldots A(x) x\right)^{1 / n} \geq \lambda(c x)>1 .
$$

In particular, $\lim _{n \rightarrow \infty} \min \left(f^{n}(x)\right)=\infty$. 
Finally, assume that $\lambda(\infty)>1$ and $\lambda(0)<1$. Since $\lambda(0)<1,0$ is an attracting fixed point with an open basin of attraction:

$$
\mathcal{B}(0)=\left\{x \in C: \lim _{n \rightarrow \infty} f^{n}(x)=0\right\} .
$$

On the other hand, the following lemma implies that $\mathcal{B}(0)$ is bounded.

Lemma 2. Assume $\lambda(0)<1$ and $\lambda(\infty)>1$. Then there exists an $M>0$ such that

$$
\lim _{n \rightarrow \infty} \min \left(f^{n}(x)\right)=\infty
$$

whenever $\|x\| \geq M$.

Proof. The definition of $\lambda(\infty)$ and the assumption that $\lambda(\infty)>1$ imply that there exists an $M_{1}$ such that $\lambda(x)>1$ whenever $\min (x) \geq M_{1}$. Let $z=M_{1}(1, \ldots, 1)$. Since $\lambda(z)>1$, the Perron-Frobenius Theorem implies that there exist an $\epsilon>0$ and $L>0$ such that $A^{n}(z) x \geq(1+\epsilon)^{n} x$ whenever $x \in C$ and $n \geq L$. Choose $1>\delta>0$ such that all entries of $A(0)^{N}$ are greater than $\delta$ and the maximum entry of each row in $A(0)$ is greater than $\delta$. (Note: $A(0)$ being primitive implies that no row vector of $A(0)$ is the zero vector.) Define $M=\frac{M_{1}}{\delta^{L+1}}$. Let $x \in C$ be such that $\|x\| \geq M$. Our choice of $x$ and $\delta$ imply that for $i=0,1, \ldots, L$,

$$
\begin{aligned}
f^{N+i}(x) & =A\left(f^{N+i-1}(x)\right) \ldots A(x) x \\
& \geq A(0)^{N+i} x \geq\|x\| \delta^{i+1}(1, \ldots, 1) \\
& \geq \frac{M_{1}}{\delta^{L-i}}(1, \ldots, 1) \geq M_{1}(1, \ldots, 1)=z .
\end{aligned}
$$

Since $f^{i}\left(f^{N} x\right) \geq z$ for $i=0, \ldots L$, our choice of $L$ implies that

$$
\begin{aligned}
f^{L+N}(x) & =A\left(f^{L-1}\left(f^{N}(x)\right)\right) \ldots A\left(f\left(f^{N}(x)\right)\right) A\left(f^{N}(x)\right) f^{N}(x) \\
& \geq A(z)^{L} z \geq(1+\epsilon)^{L} z .
\end{aligned}
$$

Thus, we may inductively conclude that

$$
f^{n+N}(x) \geq(1+\epsilon)^{n} z
$$

for $n \geq L$. In particular, $\lim _{n \rightarrow \infty} \min \left(f^{n}(x)\right)=\infty$.

The boundary $\partial \mathcal{B}(0)$ of $\mathcal{B}(0)$ is a nonempty compact invariant set for $f$. Consequently, work of Takáč [10, Prop. 1.3] implies that there exists an invariant Lipschitz hypersurface $\Gamma$ containing $\partial \mathcal{B}(0)$ that separates $C$ into two pieces. In fact, work of Terešćák [11] implies that $\Gamma$ is a continuously differentiable manifold (see also [1, in which this result is proven in the finite-dimensional case when $f$ is $C^{2}$ ).

Next, we prove that $\Gamma$ is a repellor: there exists a neighborhood $U$ of $\Gamma$ such that for all $x \in U \backslash \Gamma, f^{n}(x) \notin U$ for all $n$ sufficiently large. Since $\Gamma$ is compact and does not contain the origin, there exists a constant $c>0$ such that $c(1,1, \ldots, 1) \gg x \gg$ $(1,1, \ldots, 1) / c$ for all $x \in \Gamma$. Since $\lim _{n \rightarrow \infty} c^{1 / n}=1$ and $\Gamma$ is invariant,

$$
\lim _{n \rightarrow \infty}\left\|A\left(f^{n-1}(x)\right) \ldots A(x) x\right\|^{1 / n}=\lim _{n \rightarrow \infty}\left\|f^{n}(x)\right\|^{1 / n}=1
$$

for all $x \in \Gamma$. We need the following result of Ruelle.

Proposition 1 ([5] Prop. 3.2]). Let $X$ be a compact metric space, $f: X \rightarrow X a$ continuous map, and $T$ a continuous map from $X$ to the space of $k \times k$ nonnegative primitive matrices. Then there exist a continuous $T$-invariant splitting $E \oplus F$ of 
$X \times \mathbf{R}^{k}$ and constants $\alpha<1$ and $K \geq 0$ such that, for all $x \in X$,

- $F(x)$ is one-dimensional and contained in $\mathcal{C}=\left\{y \in \mathbf{R}^{k}: y \gg 0\right.$ or $y \ll$ 0 or $y=0\}$,

- $E(x) \backslash\{0\}$ is contained in the interior of the the complement of $\mathcal{C}$, and

- for all unit vectors $v \in E(x)$ and $w \in F(x)$,

$$
\left\|T\left(f^{n-1}(x)\right) \ldots T(x) v\right\| \leq K \alpha^{n}\left\|T\left(f^{n-1}(x)\right) \ldots T(x) w\right\| .
$$

Ruelle's result applied to $T=A$ and (2) implies

$$
\lim _{n \rightarrow \infty}\left\|A\left(f^{n-1}(x)\right) \ldots A(x) v\right\|^{1 / n}=1
$$

for all $x \in \Gamma$ and nonzero $v \in C$. By the chain rule, the derivative $D f(x)$ of $f(x)$ equals

$$
D f(x)=A(x)+D A(x) x .
$$

A3 implies that all the entries of $D A(x)$ are nonnegative. A4 implies that $D A(x)$ contains at least one positive entry for all $x \in C$. The arguments used to prove Lemma 11 imply that

$$
D f^{N}(x)=D f\left(f^{N-1}(x)\right) \ldots D f(f(x)) D f(x) \gg A\left(f^{N-1}(x)\right) \ldots A(f(x)) A(x) .
$$

Compactness and invariance of $\Gamma$ imply that there exists an $\epsilon>0$ such that

$$
D f^{n}(x) \geq(1+\epsilon)^{n} A\left(f^{n-1}(x)\right) \ldots A(f(x)) A(x)
$$

for all $x \in \Gamma$ and $n \geq N$. Equation (3) implies

$$
\liminf _{n \rightarrow \infty}\left\|D f^{n}(x) v\right\|^{1 / n} \geq 1+\epsilon
$$

for all $x \in \Gamma$ and nonzero $v \in C$. Proposition 1 applied to $T=D f$ and $X=\Gamma$ yields a $D f$-invariant splitting $E \oplus F$ in which $E$ is the tangent bundle to $\Gamma$ and $F$ is a one-dimensional vector bundle transverse to $\Gamma$. Now consider the following theorem.

Theorem 2 ([6, Thm. 1]). Let $f: X \rightarrow X$ be a continuous map of a compact metric space $X$. If $F_{n}: X \rightarrow \mathbf{R}$ is a sequence of continuous and subadditive functions (i.e., $F_{n+m}(x) \leq F_{n}(x)+F_{m}\left(f^{n} x\right)$ for all $n, m \geq 1$ and $x \in \Gamma$ ), then

$$
\sup _{x \in \Gamma} \limsup _{n \rightarrow \infty} \frac{F_{n}(x)}{n}=\inf _{n \geq 1} \frac{1}{n} \sup _{x \in \Gamma} F_{n}(x) .
$$

This theorem and equation (4) applied to $X=\Gamma$ and $F_{n}(x)=-\ln \left\|D f^{L}(x) v\right\|$ imply that there exists $L>0$ such that

$$
\left\|D f^{L}(x) v\right\| \geq(1+\epsilon / 2)^{L}\|v\| \quad \text { for } v \in F(x), x \in \Gamma .
$$

It follows that $\Gamma$ is a repellor: there exists a neighborhood $U$ of $\Gamma$ such that for all $x \in U \backslash \Gamma$ we have $f^{n}(x) \notin U$ for all $n$ sufficiently large.

To complete the proof of the theorem, it suffices to show that if $x \gg \Gamma$, then $\lim _{n \rightarrow \infty} \min \left(f^{n}(x)\right)=\infty$ and if $x \ll \Gamma$, then $\lim _{n \rightarrow \infty} f^{n}(x)=0$. We will prove the former statement, since the latter statement is proven in an analogous manner. Suppose $x \gg \Gamma$. Then there exists a $y \in \Gamma$ such that $x \gg y$. Since $\Gamma$ is a repellor, there exists an $m$ such that $f^{n}(x) \notin U$ for all $n \geq m$. Compactness of $\Gamma$ implies there exists a $\delta>0$ such that for all $n \geq m$, one entry of $A\left(f^{n} x\right)$ is at least a 
factor $(1+\delta)$ larger than the corresponding entry of $A\left(f^{n} y\right)$. The arguments used to prove Lemma 1 imply that there exists an $\epsilon>0$ such that

$$
A\left(f^{n+N}(x)\right) \ldots A\left(f^{n+1}(x)\right) \geq(1+\epsilon)^{N} A\left(f^{n+N}(y)\right) \ldots A\left(f^{n+1}(y)\right)
$$

for all $n \geq m$. Since $y \in \Gamma$, (2) implies

$$
\begin{aligned}
\limsup _{n \rightarrow \infty} \min \left(f^{n}(x)\right)^{1 / n} & =\limsup _{n \rightarrow \infty} \min \left(A\left(f^{n-1}(x)\right) \ldots A(x) x\right)^{1 / n} \\
& \geq \limsup _{n \rightarrow \infty}(1+\epsilon) \min \left(A\left(f^{n-1}(y)\right) \ldots A(y) x\right)^{1 / n}=1+\epsilon .
\end{aligned}
$$

In particular, $\lim _{n \rightarrow \infty} \min \left(f^{n}(x)\right)=\infty$.

\section{Application to age-structured models}

Consider a population that consists of $k$ distinct stages. Let $x=\left(x_{1}, \ldots, x_{k}\right)$ denote the vector of the stage densities. A standard model (known as a nonlinear Leslie matrix model [2]) for this population is given by

$$
A(x)=\left[\begin{array}{cccccc}
0 & f_{2}(x) & f_{3}(x) & \ldots & f_{k-1}(x) & f_{k}(x) \\
s_{1}(x) & 0 & 0 & \ldots & 0 & 0 \\
0 & s_{2}(x) & 0 & \ldots & 0 & 0 \\
\vdots & \vdots & \vdots & \vdots & \vdots & \vdots \\
0 & 0 & 0 & \ldots & s_{k-1}(x) & s_{k}(x)
\end{array}\right]
$$

where $f_{i}(x)$ and $s_{i}(x)$ are functions from $C$ to $[0, \infty)$ and $(0,1)$ respectively. $f_{i}(x)$ represents the average number of progeny produced from an individual in stage $i$. $s_{i}(x)$ is the fraction of individuals in stage $i$ that make it to stage $i+1$. An important quantity associated with $A(x)$ is the reproductive value $R(x)$ of a stage 1 individual:

$R(x)=s_{1}(x) f_{2}(x)+\cdots+s_{1}(x) \ldots s_{k-2}(x) f_{k-1}(x)+s_{1}(x) \ldots s_{k-1}(x) f_{k}(x) /\left(1-s_{k}(x)\right)$.

Corollary 1. Assume that

- $x \mapsto s_{i}(x)$ and $x \mapsto f_{i}(x)$ are continuously differentiable,

- $s_{i}(0)>0$ for $1 \leq i \leq k$ and $f_{i}(0)>0$ for some $i \geq 2$,

- $s_{i}(y) \geq s_{i}(x)$ and $f_{i}(y) \geq f_{i}(x)$ whenever $x \geq y$, and

- there exist $i$ and $j$ such that either $\frac{\partial s_{i}}{\partial x_{j}}(x)>0$ for all $x \in C$ or $\frac{\partial f_{i}}{\partial x_{j}}(x)>0$ for all $x \in C$.

Then:

- If $R(0) \geq 1$, then $\lim _{n \rightarrow \infty} \min \left(f^{n}(x)\right)=\infty$ for all nonzero $x \in C$.

- If $R(\infty) \leq 1$, then $\lim _{n \rightarrow \infty} f^{n}(x)=0$ for all $x \in C$.

- If $R(0)<1$ and $R(\infty)>1$, then there exists a continuously differentiable invariant hypersurface $\Gamma$ that separates $C$ into two pieces. For points $x$ below $\Gamma$,

$$
\lim _{n \rightarrow \infty} f^{n}(x)=0
$$

Alternatively, for points $x$ above $\Gamma$,

$$
\lim _{n \rightarrow \infty} \min \left(f^{n}(x)\right)=\infty .
$$

Functions that satisfy the assumptions of this corollary include $s_{i}(x)=$ $\exp \left(-b /\left(1+\beta_{1} x_{1}+\cdots+\beta_{k} x_{k}\right)\right)$ representing the fraction of individuals in stage $i$ that escape predation from a satiating generalist predator. 
Proof. The assumptions of the corollary ensure that $\mathbf{A} \mathbf{1}-\mathbf{A} \mathbf{4}$ are satisfied. To apply Theorem 1, it suffices to show that $\lambda(x)>1$ (resp. $\lambda(x)<1$ ) if and only if $R(x)>1$ (resp. $R(x)<1$ ). The characteristic polynomial of $A(x)$ is given by

$$
\begin{array}{r}
(-\lambda)^{k-1}\left(s_{k}-\lambda\right)-s_{1} f_{2}(-\lambda)^{k-3}\left(s_{k}-\lambda\right)+s_{1} s_{2} f_{3}(-\lambda)^{k-4}\left(s_{k}-\lambda\right)+\ldots \\
+(-1)^{k} s_{1} s_{2} \ldots s_{k-2} f_{k-1}\left(s_{k}-\lambda\right)+(-1)^{k+1} s_{1} \ldots s_{k-1} f_{k} .
\end{array}
$$

Setting this equal to zero and rearranging terms (including a division by $s_{k}-\lambda$, which is permissible since the dominant eigenvalue of $A(x)$ is $>s_{k}$ ) yields that the dominant eigenvalue $\lambda=\lambda(x)$ of $A(x)$ must satisfy

$$
\begin{aligned}
1= & s_{1} f_{2} / \lambda^{2}+s_{1} s_{2} f_{3} / \lambda^{3}+\cdots+s_{1} \ldots s_{k-2} f_{k-1} / \lambda^{k-1} \\
& +s_{1} \ldots s_{k-1} f_{k} /\left(\lambda^{k-1}\left(\lambda-s_{k}\right)\right) .
\end{aligned}
$$

Since $\lambda(x)>s_{k}$ and the right-hand side of (5) is a decreasing function of $\lambda$ for $\lambda>s_{k}$, we get that $\lambda(x)>1$ (resp. $\lambda(x)<1$ ) if and only if $R(x)>1$ (resp. $R(x)<1)$.

\section{REFERENCES}

1. M. Benaïm, On invariant hypersurfaces of strongly monotone maps, J. Differential Equations 137 (1997), no. 2, 302-319. MR 98d:58114

2. H. Caswell, Matrix population models, Sinauer, Sunderland, Massachuesetts, 2001.

3. F. Courchamp, T. Clutton-Brock, and B. Grenfell, Inverse density dependence and the Allee effect, TREE 14 (1999), 405-410.

4. M. P. Hassell, The dynamics of arthropod predator-prey systems, Monographs in Population Biology, vol. 13, Princeton University Press, Princeton, New Jersey, 1978. MR 80d:92026

5. D. Ruelle, Analycity properties of the characteristic exponents of random matrix products, Adv. in Math. 32 (1979), no. 1, 68-80. MR 80e:58035

6. S. J. Schreiber, On growth rates of subadditive functions for semiflows, J. Differential Equations 148 (1998), 334-350. MR 2000a:37004

7. E. Seneta, Nonnegative matrices and Markov chains, Springer-Verlag, New York, 1981. MR 85i:60058

8. P. A. Stephens and W. J. Sutherland, Conseqeuences of the Allee effect for behavior, ecology, and conservation, TREE 14 (1999), 401-405.

9. P. A. Stephens, W. J. Sutherland, and R. P. Freckleton, What is the Allee effect?, Oikos $\mathbf{8 7}$ (1999), 185-190.

10. P. Takáč, Domains of attraction of generic $\omega$-limit sets for strongly monotone discrete-time semigroups, J. Reine Angew. Math. 423 (1992), 101-173. MR 93c:58127

11. I. Tereščák, Dynamics of $C^{1}$ smooth strongly monotone discrete-time dynamical systems, preprint (1996).

Department of Mathematics, College of William and Mary, Williamsburg, Virginia 23187-8795

E-mail address: sjschr@wm.edu 